\title{
THE Z VELOCITY DISPERSION OF OUTER H I DISKS
}

\author{
JOHN M. DICKEY \\ University of Minnesota \\ 116 Church St. SE, Minneapolis, MN 55455, USA
}

\begin{abstract}
The velocity dispersion of the interstellar gas measured perpendicular to the disk in face-on spiral galaxies shows a remarkable consistency. Typically the width of the velocity distribution decreases monotonically with increasing galactic radius through the luminous, stellar disk; but in the outer disk where there is gas but no stars the velocity dispersion is $\sigma_{v} \simeq$ $7 \mathrm{~km} \mathrm{~s}^{-1}$ with a scatter of $2 \mathrm{~km} \mathrm{~s}^{-1}$ at most from one position to another and from one galaxy to another. Trying to understand this number raises questions about the thermal state of the H I in outer, gaseous disks. The most important issue is to understand the dominant heating and cooling processes for the atomic gas.
\end{abstract}

\section{Background}

The velocity distribution perpendicular to the disk of a spiral galaxy provides a useful measure of the kinematics of a population of objects. For stars in the Milky Way the relationship between the disk scale height and the $\mathrm{z}$-velocity dispersion $\left(\sigma_{v}, \mathrm{z}\right.$ being the direction perpendicular to the disk) provides a measure of the local gravitational potential, as pointed out by Oort (1932). For gas the situation is more complicated since clouds are not ballistic; the pressure of the cosmic rays and the magnetic field, which are bound to the ionized component of the gas, also contributes to the scale height (Lockman and Gehman, 1992). For other galaxies we cannot observe both the scale height and the velocity dispersion in the same system, so to determine the gravitational potential of spiral disks we must generalize over statistical samples of face-on and edge-on spirals (van der Kruit and Shostak, 1983). Observing the $\lambda 21-\mathrm{cm}$ line of neutral hydrogen in face-on galaxies we can measure the z-velocity distribution function of the inter- 
stellar atomic gas, which is of interest as a measure of the cloud velocity distribution and the temperature of the warm phase of the medium. In this contribution I discuss unsolved problems of the Milky Way, and of spiral galaxies in general, which are raised by measurements of the z-velocity profile in outer, gaseous disks.

\section{Observations}

In the luminous regions of spiral disks, where young stars are common, the interstellar gas is stirred up by stellar winds, supernova remnants, and larger scale dynamical processes associated with stars, such as spiral shocks. The effect of these processes on the interstellar gas is traced morphologically in both $\mathrm{H} \alpha$ and $\mathrm{H} \mathrm{I}$; it appears also in the velocity distribution function of the interstellar gas. In the outer disks of spirals, where there is little or no evidence for stars, the gas is much more quiescent. Its motion can be very well described by a differentially rotating disk with gas on circular orbits. Even in the most face-on galaxies, the projected velocity structure on large scales is dominated by this rotation; for example, a galaxy rotating at $250 \mathrm{~km} \mathrm{~s}^{-1}$ inclined at only $5^{\circ}$ to the line of sight will show a range of 44 $\mathrm{km} \mathrm{s}^{-1}$ in projected velocity. A telescope must have a beam small enough to resolve away the spatial variations of this projected rotation pattern in order to measure the z-velocity distribution; in practice the beam size must be less than about 30" for even the most face-on, nearby galaxies. So we have good $\lambda 21-\mathrm{cm}$ data for only a few galaxies so far, although at least two research groups have surveys of more candidates underway.

The few galaxies which have been well studied show a surprising consistency in the form of their z-velocity distributions. The basic result of all studies of face-on spirals so far published is that the z-velocity distribution of the atomic gas is very nearly a perfect Gaussian. In the inner, stellar part of the disk, this Gaussian is modified by the presence of very faint wings, roughly exponential in shape, extending several times the width of the Gaussian. (These are so weak that to see them generally requires that all spectra in an annulus be shifted to a common center velocity and then added. The velocity offset for each position is determined from the model rotation field as described by Dickey, Hanson and Helou, 1990.) In the outer, dark portion of the disk no such wings have been detected; there the Gaussian fit to the mean emission profile is nearly perfect. The widths of the fitted profiles decrease with increasing radius through the stellar disk, but beyond a radius where the blue surface brightness is about 25 mag $(\operatorname{arcsec})^{-2}$ the $\mathrm{H}$ I linewidths remain constant at $\sigma_{v} \simeq 7 \mathrm{~km} \mathrm{~s}^{-1}$. This value seems to be a very general quantity, many different face-on spirals in different environments show similar behavior, with outer disk z-velocity 


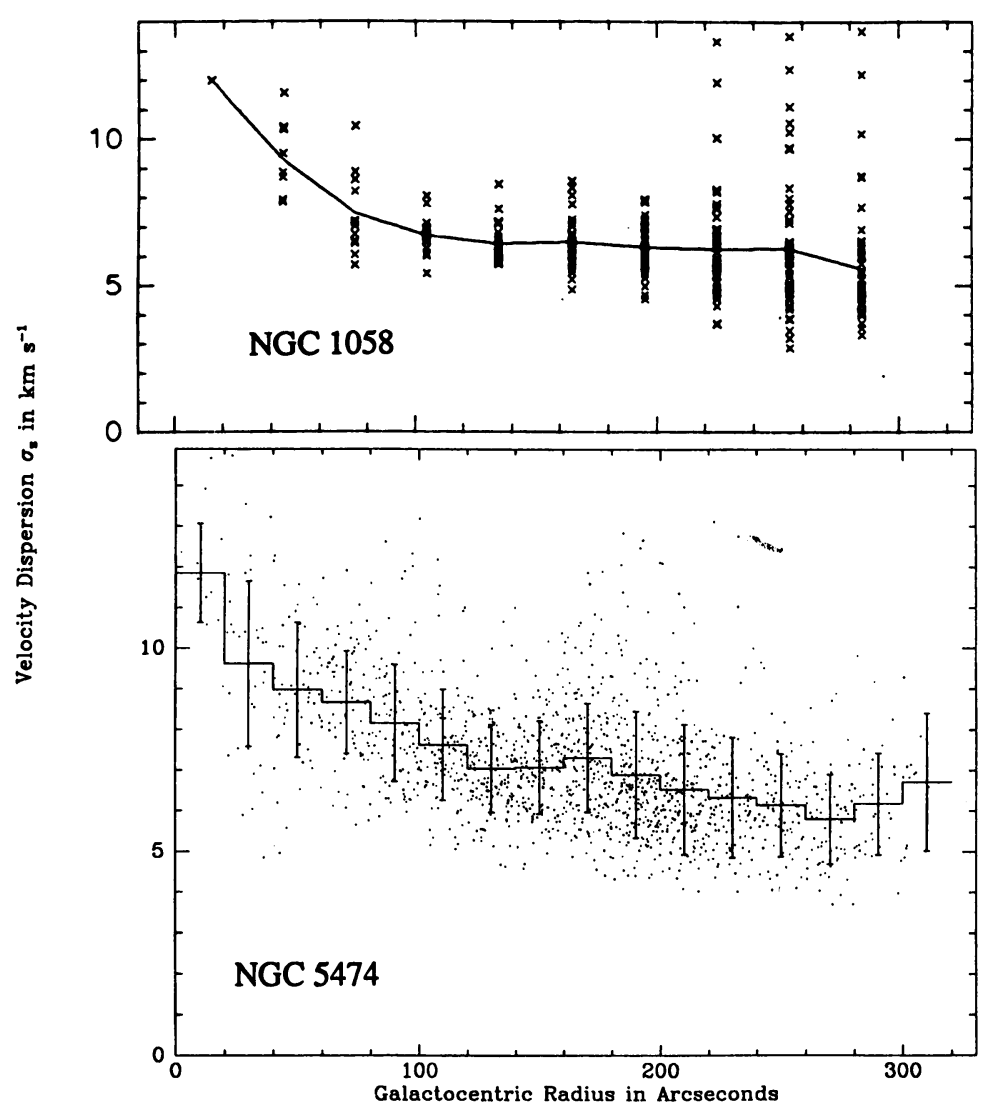

Figure 1. Examples of the radial variation of the $\mathrm{H}$ I linewidth, $\sigma_{v}$ in two face-on galaxies. The top panel shows data for NGC1058 (Dickey, Hanson, and Helou, 1990), the lower panel for NGC5474 (Rownd, Dickey, and Helou, 1994). Symbols mark the results of Gaussian fits to independent beam areas in each annulus, while the line segments join the mean values for each radius.

dispersions in the range 6 to $9 \mathrm{~km} \mathrm{~s}^{-1}$ (Rownd, Dickey and Helou, 1994; Boulanger and Viallefond, 1992). Two examples are shown on figure 1.

\section{Interpretation}

This behavior of the velocity distribution is not hard to understand in general terms. The radial variation of $\sigma_{v}$ in the stellar disks reflects the stirring of the interstellar medium by supernovae and stellar winds; the non-Gaussian tail of the velocity distribution function corresponds to the galactic fountain process (Bregman 1980, Norman and Ikeuchi 1988, Heiles 1990). A similar intermediate velocity cloud population is seen in the solar neighborhood of the Milky Way (Kulkarni and Fich 1985, Wakker 1991). In 


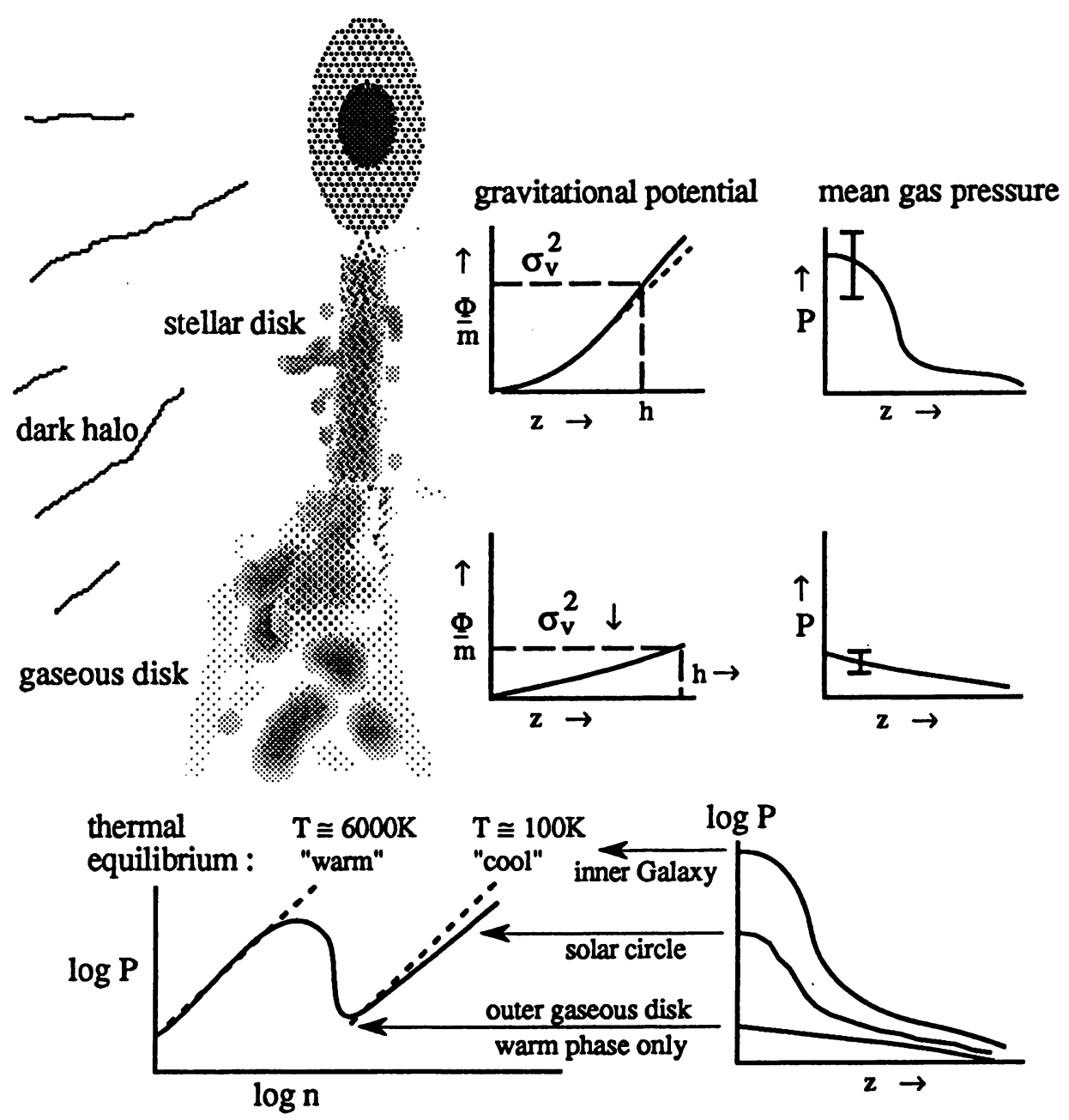

Figure 2. A schematic cross section of a spiral galaxy disk. As explained in the text, gas in the stellar disk probably has a very different pressure environment than gas in the outer part of the disk where there are no stars.

the outer disk, where there are no stars to inject turbulent kinetic energy, $\sigma_{v}$ is smaller, and there is no intermediate velocity tail. It remains a question why the value of $7 \mathrm{~km} \mathrm{~s}^{-1}$ is so common, this must reflect the outcome of some physical process at work in the outer disks to regulate $\sigma_{v}$.

A possible explanation for the ubiquity of the $7 \mathrm{~km} \mathrm{~s}^{-1}$ value for $\sigma_{v}$ is 
sketched on figure 2. The upper part of this figure shows a cartoon of how the changing gravitational potential of the disk, as a function of $z$, influences the time-averaged interstellar pressure. On the left is a schematic crosssection of a galactic disk. Next to this are two graphs of the gravitational potential vs. $z$; plotted here is the potential per unit projectile mass, $\phi / m$, whose derivative is the gravitational acceleration, $\mathrm{K}_{z}$. In the inner disk the dynamics are dominated by disk stars, so the gravitational potential is parabolic in $z$ near the midplane, where the density is roughly constant, and changes to a linear increase with $\mathrm{z}$ high above the plane where the mass density of disk stars is negligable. Even at high $\mathrm{z}$ there is some mass density due primarily to the dark matter halo, so the potential rises slightly faster than linearly; the disk alone would give something like the short-dashed line. The scale height, $\mathrm{h}$, is set by the velocity dispersion, $\sigma_{z}$; for gas this is modified by magnetic fields and cosmic rays, a factor of $(1+\alpha+\beta)$ in the notation of Spitzer (1977).

The time-average pressure of the interstellar gas must reflect the $z$ dependence of the gravitational potential, as suggested in the right-hand plots. At any point, the pressure fluctuates as a function of time due to the occasional passage of supernova remnants, spiral density waves, and other processes, hence the large error bar on the pressure graph in the inner disk. But the time-average pressure must drop with $\mathrm{z}$ roughly as the potential rises, simply to keep the system in hydrostatic equilibrium on long time scales.

In the outer disk the gravitational potential is much weaker, the gas is only weakly self-gravitating in $\mathrm{z}$ and the dark halo does not help to confine it to a plane. As a result the gaseous disk warps and flares. The gravitational potential rises so slowly with $z$ that even though the velocity dispersion is much less than in the stellar disk, the scale height is greater. The pressure must be much lower in this region, and, in the absence of stars to stir it up, there is very little fluctuation in the pressure as a function of time.

At the bottom of figure 2 is a sketch of how the pressure determines the thermal phase of the atomic medium. On the left is a plot of the heatingcooling equilibrium, in the style of Field, Goldsmith and Habing (1969). The solid line sketches the form of a typical equilibrium curve; thermally stable points correspond to positive slopes of this line, which are near $\mathrm{T}$ $\simeq 6000 \mathrm{~K}$ (the warm phase) or $\mathrm{T} \leq \sim 100 \mathrm{~K}$ (the cool phase). In the solar neighborhood, at low $\mathrm{z}$, the average pressure is in the range where both phases can coexist, but at higher $\mathrm{z}$ where the pressure is lower only the warm phase is a possible equilibrium state. In the outer galaxy the pressure may be below the minimum for which the cool phase can exist even at midplane, so we might expect that all the gas in outer, gaseous disks of spiral galaxies should be in the warm phase. 


\section{Questions}

The values of $\sigma_{v}$ that are actually measured are uncomfortably low for a disk of gas in the warm phase. If this one dimensional velocity dispersion is translated directly to a thermal velocity, then $7 \mathrm{~km} \mathrm{~s}^{-1}$ gives kinetic temperature $\mathrm{T}_{k i n}=5900 \mathrm{~K}$, which is reasonable. However there are many other possible contributions to the linewidth besides microscopic thermal motions; these include both instrumental effects such as beam smearing of the projected rotation field and finite spectrometer channel widths as well as dynamical processes in the gas, such as cloud motions, shocks, and random departures from circular orbital motion. In the Milky Way where we can measure separately the kinetic temperature and the velocity dispersion of the gas in some regions we typically find that the total linewidth is about twice the contribution from thermal motions alone. These considerations suggest that a linewidth of $7 \mathrm{~km} \mathrm{~s}^{-1}$ should correspond with a kinetic temperature of about 1500 to $2000 \mathrm{~K}$, which is low for a stable thermal equilibrium unless the heating and cooling processes are quite different from what we are familiar with in the solar neighborhood.

A more vague question concerns the role of cool, diffuse clouds in outer gaseous disks. There must be some H I in the cool phase in outer disks, as there are isolated spots of star formation and occasionally molecular clouds are seen even well outside the optical disks of spiral galaxies. The $\sigma_{v}$ for a population of cool clouds will be determined primarily by the random velocity distribution of the clouds, as the thermal linewidth for gas at $100 \mathrm{~K}$ is slightly less than one $\mathrm{km} \mathrm{s}^{-1}$. If a significant amount of the outer disk gas is in the form of clouds, then it is hard to understand the constancy of the value $\sigma_{v} \simeq 7 \mathrm{~km} \mathrm{~s}^{-1}$, unless there is some physical process which regulates the cloud random velocity distribution. But such a process must work only in outer disks, since we know that in stellar disks the cloud population has a tail in velocity reaching much higher than $7 \mathrm{~km} \mathrm{~s}^{-1}$.

In conclusion, I feel that there are a lot of unsolved problems associated with the outer, gaseous disks of spiral galaxies. The dark matter which dominates their large scale dynamics is a problem in itself. On smaller scales, the $\mathrm{z}$ velocity distribution raises issues of cooling, heating, and ionization, some of which have been discussed in other talks at this meeting. As new telescopes come on-line with greatly improved sensitivity and resolution, particularly for observing tracers of the interstellar medium in the radio, millimeter-wave, sub-millimeter, and infrared bands, we may hope in the next decade to vastly expand our knowledge of the astrophysics of the gas in the outer, dark regions of spiral disks. 


\section{References}

Boulanger, F. and Viallefond, F. (1992), Astron. Astrophys. 266, 37.

Bregman, J.N. (1980), Ap. J. 236, 577.

Dickey, J.M., Hanson, M.M., and Helou, G. (1990), Ap. J. 352, 522.

Heiles, C. (1990), Ap. J. 354, 483.

Kulkarni, S.R. and Fich, M. (1985), Ap. J. 289, 792.

Lockman, F.J. and Gehman, C., (1991), Ap. J. 382, 182.

Norman, C.A. and Ikeuchi, S. (1989), Ap.J. 345, 372.

Oort, J.H. (1932), Bull. Astron. Inst. Neth. 6, 249.

Rownd, B.K., Dickey, J.M., and Helou, G. (1994), A.J. 108, 1638.

Spitzer, L. Jr. (1977), Physical Processes in the Interstellar Medium, (New York : John Wiley), chap. 11.

van der Kruit, P., and Shostak, S. (1984), Astron. Astrophys., 134, 258.

Wakker, B. (1991), Astron. Astrop. 250, 499.

\section{DISCUSSION}

Shull: I believe that if one goes far enough out in the disk, the metagalactic radiation field can provide the heating necessary for $\sigma_{v} \simeq 7 \mathrm{~km} \mathrm{~s}^{-1}$. (This may require dust in this $\mathrm{H} \mathrm{I}$, for photoelectric heating.)

Dickey: Yes, then the metagalactic radiation field is crucial not only for ionizing the outer edges of gas disks, but also for heating the regions which remain neutral. 